Main Outcome Measurements The primary outcome was the overall injury prevalence (\%) measured every fortnight with the online Oslo Sports and Trauma Research Centre questionnaire. Secondary outcome scores included prevalence of substantial injuries, overall incidence, time-loss injuries, exposure, adherence and experiences.

Results The mean injury prevalence was 23\% (95\% CI 20-26) in the IPPON group and $28 \%$ in the control group (95\% CI 25-30). The risk of reporting injuries was 18\% lower in the IPPON group (OR 0.72 95\% CI $0.37-1.39$, adjusted p-value of 0.33 ). Secondary outcome scores showed no differences between groups. For substantial injuries there was a $22 \%$ lower risk in the IPPON group (OR 0.80, 95\% CI 0.36-1.78, adjusted p-value 0.58). Trainers and athletes experienced the IPPON intervention as successful.

Conclusions The IPPON trainer-supervised judo-specific injury prevention programme did not reach statistical significance in reducing the overall injury prevalence. The best-estimate of $18 \%$ injury reduction rate and successful experience indicate that the IPPON intervention might be practicable and relevant for the judo community.

\section{DOES INCREASING THE SEVERITY OF PENALTIES ASSESSED IN ASSOCIATION WITH THE 'ZERO TOLERANCE FOR HEAD CONTACT' POLICY TRANSLATE TO A REDUCTION IN HEAD IMPACT RATES IN YOUTH ICE HOCKEY?}

${ }^{1}$ Rylen A Williamson, ${ }^{1}$ Ash T Kolstad, ${ }^{6}$ Luc Nadeau ${ }^{6}{ }^{6}$ Claude Goulet, $1,2,3,4,5$ Brent Hagel, $1,2,3,4,5$ Carolyn A Emery. 'Sport Injury Prevention Research Centre, University of Calgary, Calgary, Canada; ${ }^{2}$ Alberta Children's Hospital Research Institute, Calgary, Canada; ${ }^{3}$ Departments of Paediatrics, Cumming School of Medicine, University of Calgary, Calgary, Canada; ${ }^{4}$ Department of Community Health Sciences, Cumming School of Medicine, University of Calgary, Calgary, Canada; ${ }^{5} \mathrm{O}^{\prime}$ Brien Institute for Public Health, Cumming School of Medicine, University of Calgary, Calgary, Canada; ${ }^{6}$ Department of Physical Education, Faculty of Education, Université Laval, Québec City, Canada

10.1136/bjsports-2021-IOC.203

Background The risk of concussion is high in Canadian youth ice hockey. Aiming to reduce this burden, in 2011 Hockey Canada implemented a national 'zero tolerance for head contact (HC)' policy mandating the penalization of any player-toplayer HC. In 2018-20, Hockey Canada further amended this HC-policy including stricter enforcement of severe HCs.

Objective To compare HC rates and HC enforcement pre-policy, post-policy, and following policy amendments in elite U15 Canadian youth ice hockey.

Design Prospective cohort.

Setting A collection of events recorded with a video-camera located at the highest point near centre-ice in public ice hockey arenas in Calgary, Alberta.

Participants A convenience sample of 10 AA (elite) U15 (1314 year old) games pre-policy (2008-09), 8 games post-policy (2013-14), and 10 games following policy amendments (2020-21).

Assessment of Risk Factors An analysis of HC-policy implementation and policy amendments across three cohort years.

Main Outcome Measurements Using Dartfish video-analysis software; all player contacts and HCs [direct (HC1), indirect (e.g., boards, ice) (HC2)] were tagged using validated criteria. Univariate Poisson regression [clustering by team-game offset by game-length (minutes)] was used to estimate HC1 and HC2 incidence rates (IR) and incidence rate ratios (IRR) between cohorts.
Results A total of 11,427 physical contacts were tagged $\left(\mathrm{n}_{2008-09}=3896, \mathrm{n}_{2013-14}=3183, \mathrm{n}_{2020-21}=4348\right)$, with 538 contacts including the head $\left(340\right.$ HC1,198 HC2) $\left(\mathrm{n}_{2008-}\right.$ ${ }_{0} \mathrm{HC} 1=125, \mathrm{HC} 2=66 ; \mathrm{n}_{2013-14} \mathrm{HC} 1=110, \mathrm{HC} 2=44 ; \mathrm{n}_{2020}$ ${ }_{21} \mathrm{HC} 1=105, \mathrm{HC} 2=88$ ). With additional rule modifications, a $30 \%$ reduction in $\mathrm{HC} 1 \mathrm{~s}$ emerged $\left(\mathrm{IRR}_{2013-2020}=0.70\right.$, 95\%CI:0.51-0.95). Since the HC-policy implementation, HC1s decreased by $24 \%\left(\mathrm{IRR}_{2008-2020}=0.76\right.$, 95\%CI:0.580.99). The proportion of $\mathrm{HC} 1 \mathrm{~s}$ penalized was similar across cohorts $\left(\mathrm{P}_{2008-09}=14.4 \% ; \mathrm{P}_{2013-14}=15.5 \% ; \mathrm{P}_{2020-}\right.$ $\left.{ }_{21}=16.2 \%\right)$.

Conclusions The HC-policy amendments and increased policy implementation time have led to a decreased rate of HC1s. However, referee enforcement can further boost the HC-policy effectiveness. These findings can help future referee training and potential rule modifications to increase player safety nationally.

\section{INJURIES IN PORTUGUESE RECREATIONAL SURFERS}

${ }^{1,6}$ Maria António Castro, ${ }^{2}$ Rodrigo Fernandes, ${ }^{3,4}$ Mário A Rodrigues-Ferreira, ${ }^{5}$ João Madail, ${ }^{3,4}$ António VencesBrito. ${ }^{1}$ School of Health Sciences, Polytechnic of Leiria, Leiria, Portugal; ${ }^{2}$ Physiotherapy Department of Coimbra Health School - Instituto Politécnico de Coimbra, Portugal, Coimbra, Portugal; ${ }^{3}$ Sport Sciences School of Rio Maior, Polytechnic Institute of Santarém, Rio Maior, Portugal; ' ${ }^{4}$ Life Quality Research Center - CIEQV, Rio Maior, Portugal; ${ }^{5}$ FisioCentro - Gabinete de Fisioterapia de Albergaria-a-Velha, Aveiro, Portugal; ${ }^{6}$ Centre for Mechanical Engineering, Materials and Processes (CEMMPRE), University of Coimbra, Coimbra, Portugal

\subsection{6/bjsports-2021-IOC.204}

Background Surfing practice has been growing in recent years with an increasing number of recreational practitioners, especially in countries like Portugal with very good ocean conditions. Most studies consider all professional and recreational surfer injuries.

Objective Determine the rate of injuries and their characteristics during the recreational surf practice.

Design Retrospective cohort study.

Setting Standardised data collected with a validated questionnaire.

Patients 150 Portuguese surfers aged between 8 to 68 years practising during at least one of the two seasons in the study agreed to participate.

Interventions Both gender recreational surfers.

Main Outcome Measurements Retrospective assessment of the last 2 years injuries occurrence and conditions of occurrence.

Results A total of 33 athletes sustained 45 injuries (22\% injured players) with 9,72 injuries per $1000 \mathrm{~h}$ of exposure. The greatest number of injuries occurred in the lower leg (ankle 20\%; knee 13\%) followed by the shoulder (17.8\%). The most common injury mechanism was collision/direct contact $(59 \%)$ or torsion $(11.4 \%)$ with the joints most affected (24.4\%). Injuries more frequent were wound $(17.8 \%)$, contusion $(11 \%)$ and inflammation (11\%). The surfboard $(28.9 \%)$ and the athlete itself (24.4\%) were the main cause of the injury, occurring mainly when performing manoeuvres $(20.9 \%)$ especially descending the wave $(16.3 \%)$. There are no statistically significant differences in injury frequency per $1000 \mathrm{~h}$ of exposure with regards to sex, surfer position (goofy or regular), surfing side (left, right or both). Male athletes demonstrate higher injury rates (women 7.94, SD 1.96; men 10.27, SD 2.89) per $1000 \mathrm{~h}$ of exposure. 